\title{
Blood pressure variability and cardiovascular disease: systematic review and meta-analysis
}

\author{
Sarah L Stevens, ${ }^{1}$ Sally Wood, ${ }^{1}$ Constantinos Koshiaris, ${ }^{1}$ Kathryn Law, ${ }^{1}$ Paul Glasziou, ${ }^{2}$ Richard J Stevens, \\ Richard J McManus ${ }^{1}$
}

${ }^{1}$ Nuffield Department of Primary Care Health Sciences, University of Oxford, Radcliffe Observatory Quarter, Oxford OX2 6GG, UK

${ }^{2}$ Faculty of Health Sciences and Medicine, Bond University,

Queensland, Australia

Correspondence to: RJ Stevens richard.stevens@phc.ox.ac.uk Additional material is published online only. To view please visit the journal online.

Cite this as: BMJ 2016;354:14098 http://dx.doi.org/10.1136/bmj.i.4098

Accepted: 02 July 2016

\section{ABSTRACT \\ OBJECTIVE \\ To systematically review studies quantifying the associations of long term (clinic), mid-term (home), and short term (ambulatory) variability in blood pressure, independent of mean blood pressure, with cardiovascular disease events and mortality. \\ DATA SOURCES \\ Medline, Embase, Cinahl, and Web of Science, searched to 15 February 2016 for full text articles in English.}

\section{ELIGIBILITY CRITERIA FOR STUDY SELECTION}

Prospective cohort studies or clinical trials in adults, except those in patients receiving haemodialysis, where the condition may directly impact blood pressure variability. Standardised hazard ratios were extracted and, if there was little risk of confounding, combined using random effects meta-analysis in main analyses. Outcomes included all cause and cardiovascular disease mortality and cardiovascular disease events. Measures of variability included standard deviation, coefficient of variation, variation independent of mean, and average real variability, but not night dipping or day-night variation.

RESULTS

41 papers representing 19 observational cohort studies and 17 clinical trial cohorts, comprising 46 separate analyses were identified. Long term variability in blood pressure was studied in 24 papers, mid-term in four, and short-term in 15 (two studied both long term and short term variability). Results from 23 analyses were excluded from main analyses owing to high risks of confounding. Increased long term variability in systolic blood pressure was associated with risk of all cause

\section{WHAT IS ALREADY KNOWN ON THIS TOPIC}

It is well established that patients with high blood pressure are at higher risk of future cardiovascular disease

Some studies have also suggested that patients with higher variability in blood pressure over time are at higher risk compared with patients with the same mean blood pressure level

It is not clear whether this risk depends on the method of measurement of variability, and few have correctly accounted for mean blood pressure or changes in treatment

\section{WHAT THIS STUDY ADDS}

Methodological errors are present in approximately half of prospective studies of blood pressure variability, but the association of long term (clinic) variability in blood pressure with future cardiovascular disease is found even in studies that avoid errors Mid-term and short term variability in blood pressure measured at home or by ambulatory monitoring, respectively, has been little studied comparatively, but shows similar associations with outcomes

mortality (hazard ratio 1.15, 95\% confidence interval 1.09 to 1.22$)$, cardiovascular disease mortality (1.18, 1.09 to 1.28$)$, cardiovascular disease events $(1.18,1.07$ to 1.30$)$, coronary heart disease (1.10, 1.04 to 1.16$)$, and stroke $(1.15,1.04$ to 1.27$)$. Increased mid-term and short term variability in daytime systolic blood pressure were also associated with all cause mortality $(1.15,1.06$ to 1.26 and $1.10,1.04$ to 1.16 , respectively).

\section{CONCLUSIONS}

Long term variability in blood pressure is associated with cardiovascular and mortality outcomes, over and above the effect of mean blood pressure. Associations are similar in magnitude to those of cholesterol measures with cardiovascular disease. Limited data for mid-term and short term variability showed similar associations. Future work should focus on the clinical implications of assessment of variability in blood pressure and avoid the common confounding pitfalls observed to date.

\section{SYSTEMATIC REVIEW REGISTRATION} PROSPERO CRD 42014015695.

\section{Introduction}

Blood pressure is a leading risk factor for cardiovascular disease. ${ }^{12}$ Most studies have used mean blood pressure as the indicator of risk, measured in clinic or "out of office" settings. ${ }^{3-5}$ However, blood pressure shows noticeable oscillations over the short and long term. ${ }^{6}$ Historically, variability in blood pressure has been viewed as inhibiting accurate measurement of mean blood pressure and as a phenomenon to be overcome by improved monitoring. ${ }^{7}$ For at least two decades, this variability has also been recognised as a potential risk factor in its own right. ${ }^{89}$ In 2010 an analysis of three cohort studies and two randomised trials found that long term variability in blood pressure was a predictor of stroke and coronary events in high risk patients. ${ }^{10}$

However, understanding this variability has been hampered by statistical and clinical methodological problems. Some analyses of variability have not adjusted for mean blood pressure, potentially confounding high variability with high mean blood pressure, ${ }^{11}$ or have adjusted for a mean that is not fully consistent with the variability measure. ${ }^{12}$ Others, in using 24 hour mean to adjust for daytime variability, might have turned high daytime variability into a surrogate marker for nocturnal or 24 hour blood pressure. ${ }^{13}$ Further studies have defined variability on the basis of measurements taken during follow-up, but analysed it as a baseline risk factor, ${ }^{14-16}$ potentially introducing problems of informative censoring or immortal time bias. ${ }^{17}$ Informative censoring occurs when reasons for loss to follow-up are confounded with the exposure 
(eg, if individuals with extreme or erratic blood pressures are withdrawn from studies because of concerns about safety). Immortal time bias can occur if individuals are required to have a certain number of blood pressure measurements in order to be included in analysis for mortality outcomes. The time up until the qualifying measurement becomes "immortal time," because, by definition, death could not occur earlier.

Other studies failed to use consistent blood pressure monitoring equipment over time, to define a consistent measurement protocol, or to account for change in drugs, leaving doubt as to the source of any observed variability. ${ }^{141819}$ Measurement at different times of the day $^{20}$ or year, ${ }^{21}$ in different arms, ${ }^{22}$ or using inconsistent cuff sizes $^{23}$ can affect accurate measurement, thereby inducing variability. We reviewed prospective studies in adults that quantified the associations of blood pressure variability with cardiovascular events and mortality, independent of mean blood pressure. Our main analysis focused on studies meeting prespecified methodological criteria, so that any apparent effect of variability was likely to be a true independent effect.

\section{Methods}

Study selection

We searched Medline, Embase, Cinahl, and Web of Science to 15 February 2016 for full text articles in English describing trials and prospective cohort studies in adults that assessed the association of periods of variability in blood pressure with cardiovascular outcomes (see supplementary table e1). Long term variability was measured through clinic blood pressure monitoring, mid-term through home monitoring, and short term through ambulatory monitoring. Studies included in recent systematic reviews ${ }^{24-27}$ were also screened. Two reviewers (SW/SS and KL/KC) scrutinised the titles and abstracts, with adjudication by a third reviewer (RM).

\section{Inclusion and exclusion criteria}

Studies had to consider at least one of the following outcomes: all cause mortality, cardiovascular events (including stroke, myocardial infarction, coronary heart disease, and heart failure), or cardiovascular mortality (including sudden death). We excluded studies only assessing intermediate outcomes (eg, arterial intima media thickness) or concerning nocturnal dipping or day-night variation, as these have been considered previously. ${ }^{28}$

Studies in disease specific populations (eg, people with diabetes) were included, except those in patients receiving haemodialysis where changes in blood pressure (intradialysis hypotension and hypertension ${ }^{2930}$ ) are common and have been shown to be associated with hospital admission and mortality. ${ }^{31} 32$

Included studies had at least 2500 person years of follow-up. Blood pressure variability was assessed in the long term (in clinics), mid-term (at home), or short term (through ambulatory monitoring). Studies of clinic monitoring had to measure visit-to-visit variability over at least five clinic visits. Studies of home monitoring had to consider day-by-day variability over at least 12 measurements on at least three days. ${ }^{33}$ Studies for ambulatory monitoring had to assess variability up to 24 hours, with at least 14 daytime readings. ${ }^{33}$

\section{Data extraction}

Using prespecified forms, two reviewers (SS/SW and $\mathrm{KL} / \mathrm{RM}$ ) independently extracted data on study and patient characteristics and two (SS and KC/RS) on statistical results (see supplementary table e2). Hazard ratios were extracted for every variability measure and outcome. The hazard ratio from the analysis with the greatest adjustment for confounders but containing only a single variability measure was extracted. Where required data were not available, we emailed the study authors.

\section{Data analysis and statistical methods}

Hazard ratios were converted to standardised hazard ratios, using a general method for regression models (see supplementary table e3). ${ }^{34}$ Briefly, a standardised log-hazard ratio was calculated as the log-hazard ratio for each unit of standardised blood pressure variability (blood pressure variability divided by its sample standard deviation). These were pooled using a random effects meta-analysis, stratified by outcome. Separate analyses were performed for each period of variability (long term, mid-term, or short term). Heterogeneity was assessed using the $\chi^{2}$ test and $\mathrm{I}^{2}$ statistic.

Where studies used multiple measures of variability, we included hazard ratios in analysis according to the following hierarchy (preferred to least preferred): standard deviation, coefficient of variation, variation independent of mean, average real variability, standardised residual, root successive variance, and other. Where hazard ratios were calculated using data from the same primary study but reported in different papers, we included the most recently published hazard ratio. We combined the hazard ratios for study subgroups before inclusion.

Two reviewers (SS and RS) independently assessed the risk of bias using the QUIPS tool, ${ }^{35}$ with adjudication by a third reviewer (RM). We also extracted information about other potential confounders, specific to studies of blood pressure variability (see supplementary table e2). Consistency of blood pressure measurement with respect to device, cuff size, staff, and measurement is important to prevent inducing the variability. The impact of other potential confounders may be adjusted for during analyses. We decided (a priori) to include in main analyses only hazard ratios that were correctly adjusted for the equivalent mean blood pressure level (eg, adjusted for mean daytime systolic blood pressure if variability was assessed for daytime systolic blood pressure), where outcome ascertainment took place after the blood pressure measurement period and, for studies involving antihypertensive treatment, where at least $80 \%$ of patients were adherent to treatment or did not change drugs during the measurement period, or where patients were censored at the point of change of treatment. We carried out secondary analyses including all studies. 


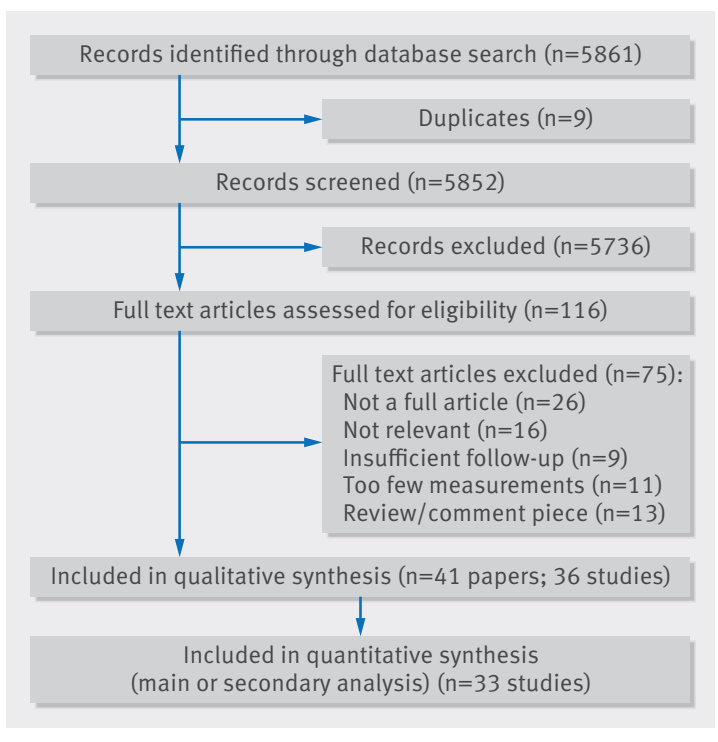

Fig 1 Study screening flowchart

Publication bias was assessed by Egger's test. ${ }^{36}$ However, since this has low power for small numbers of studies, we also calculated the number of null effect studies of mean weight that would need to be included in meta-analyses to result in a non-significant pooled effect (known as fail-safe N). ${ }^{37}$

\section{Patient involvement}

Two lay representatives contributed to the design and content of the National Institute for Health Research programme grant from which this work arose. Results from this work have been presented as part of the wider programme at regular steering group meetings.

\section{Results}

Searches identified 5861 references. Removal of duplicates and screening by two reviewers yielded 41 full text

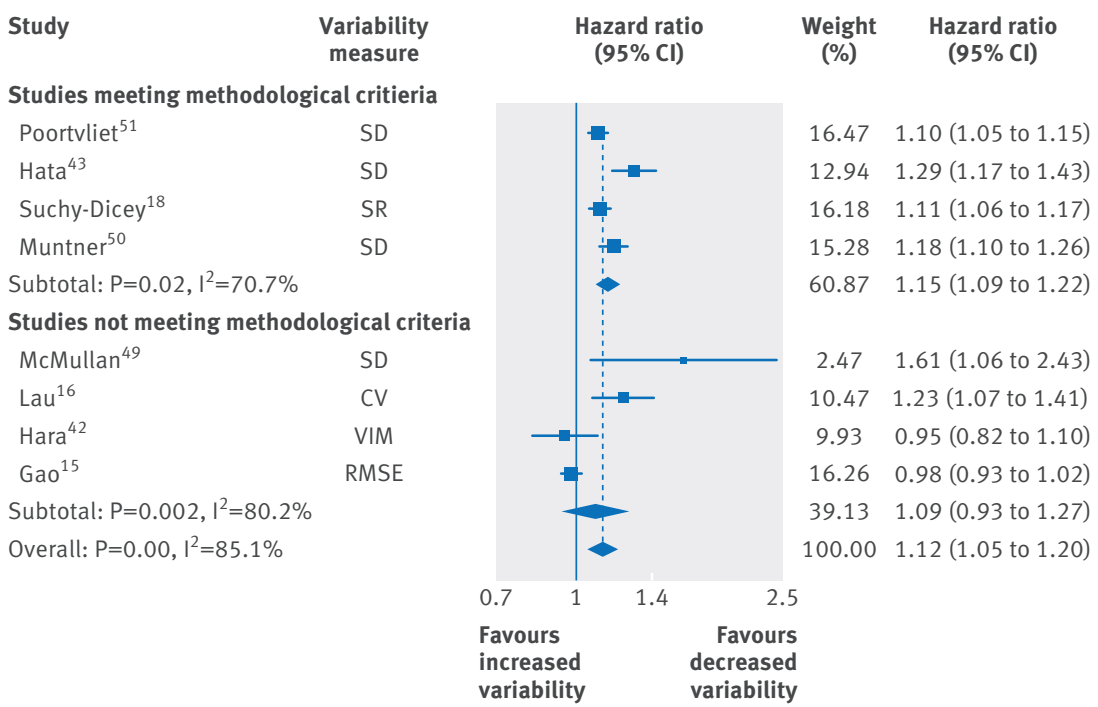

Fig 2 | Random effects meta-analysis of standardised hazard ratios for increases in clinic systolic blood pressure variability and all cause mortality. $S D=$ standard deviation; $\mathrm{SR}=$ standardised residual; $\mathrm{CV}=$ coefficient of variation; $\mathrm{VIM}=$ variation independent of the mean; RMSE=root mean squared error articles for inclusion (fig 1). These 41 papers represented 19 observational cohort studies and 17 clinical trial cohorts, and 46 separate analyses (see supplementary table e4). Twenty four papers ${ }^{1014-16181938-55}$ studied long term variability (ie, monitoring of blood pressure in clinics), four ${ }^{56-59}$ studied mid-term variability (home monitoring), and 150-1341 60-69 studied short term variability (ambulatory monitoring). The number of participants in each study ranged from $457^{41}$ to $122636^{54}$ and follow-up ranged from 2514 person years ${ }^{41}$ to 490544 person years. ${ }^{54}$

\section{Study design and analysis characteristics}

Consistency of blood pressure measurement with respect to cuff size, arm, device, and staff was unclear or had the potential to introduce variability (eg, mercury sphygmomanometers and changing staff) in all of the 36 included studies (see supplementary table e5). Similarly, the potential for confounding was introduced because of the analysis (or this was unclear) in all 46 separate analyses. Results from 23 analyses were excluded from our main analyses on the basis of the three prespecified criteria: eight analyses failed to correctly adjust for mean blood pressure, 15 did not account for major drug change during the measurement period, and 20 did not separate the measurement and follow-up periods. Results from four analyses (three studies) were not reported in sufficient detail to allow data extraction.

\section{QUIPS risk of bias}

Using QUIPS, most of the 46 analyses were rated at moderate risk of bias for study participation, often because of inclusion criteria based on blood pressure readings and a potential for regression to the mean effects (see supplementary table e6). Eighteen analyses were at high risk of bias because the measurement period for blood pressure variability was confounded by follow-up ( $\mathrm{n}=17)$, and one analysis ${ }^{67}$ failed to report non-significant results. All of the analyses rated at high risk of bias using QUIPS were excluded from our main analysis based on the assessments in supplementary table e5.

\section{Long term variability measured by clinic monitoring} Twenty four papers reported results from 27 studies that measured blood pressure variability in clinics (long term). Results from three studies ${ }^{44} 4852$ were not presented in sufficient detail for extraction.

Eight studies examined long term variability in systolic blood pressure and all cause mortality, of which four had sufficiently low risk of bias to be included in the main analysis (fig 2, standardised hazard ratio 1.15, 95\% confidence interval 1.09 to 1.22 ). Heterogeneity between studies $\left(\mathrm{I}^{2}=70.7 \%, \mathrm{P}=0.02\right)$ was reduced after removal of a study in patients with previous stroke or vascular disease: ${ }^{43}$ this did not significantly alter the results (hazard ratio 1.12, 95\% confidence interval 1.08 to $1.16 ; \mathrm{I}^{2}=34.9 \%, \mathrm{P}=0.21$ ).

Three studies assessing blood pressure variability and cardiovascular disease mortality showed a 
significant relation (see supplementary figure e1, hazard ratio $1.18,95 \%$ confidence interval 1.09 to 1.28 ) but only a single study examining cardiovascular disease events was suitable for inclusion (see supplementary figure e2, 1.18, 1.07 to 1.30).

Fourteen studies reported results for stroke events, of which six were included in the main analysis (fig 3; 1.15, 1.04 to $\left.1.27 ; \mathrm{I}^{2}=82.1 \%, \mathrm{P}<0.001\right)$. Results were similar after omission of the hazard ratio from the UK-TIA trial, ${ }^{70}$ which removed the heterogeneity $(1.10,1.05$ to $1.14 ; \mathrm{I}^{2}=0.0 \%, \mathrm{P}=0.62$ ).

Results for coronary heart disease events and myocardial infarction showed similar results (see supplementary figures e3 and e4). Across all outcomes, secondary analysis including results from all studies regardless of risk of bias did not alter results.

\section{Mid-term variability measured by home monitoring}

Four papers reported results from two studies that measured mid-term variability in home blood pressure monitoring. All four papers were of sufficient quality to be included in main analyses, but a lack of data from distinct studies meant it was only possible to perform formal meta-analysis for the all cause mortality outcome. Variability in systolic blood pressure was a significant predictor of death when blood pressure was measured in the morning or evening, or both (fig 4, eg, hazard ratio for increases in combined blood pressure variability 1.15 , 95\% confidence interval 1.06 to 1.26 ). Study level results for other outcomes are given in supplementary table e7).

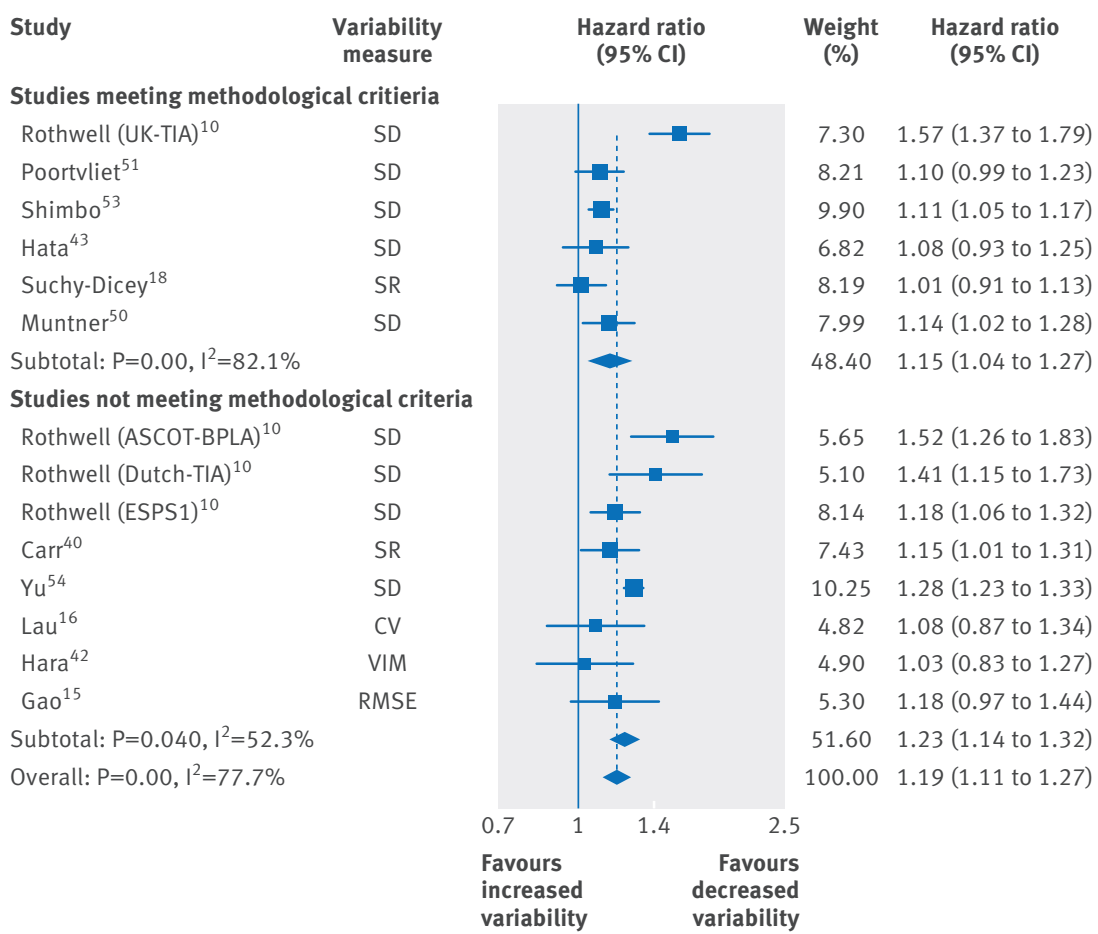

Fig 3 | Random effects meta-analysis of standardised hazard ratios for increases in clinic systolic blood pressure variability and stroke events. SD=standard deviation; $\mathrm{SR}=$ standardised residual; $\mathrm{CV}=$ coefficient of variation; $\mathrm{VIM}=$ variation independent of the mean; RMSE=root mean squared error

\section{Short term variability measured by ambulatory} monitoring

Fifteen papers examined short term variability in ambulatory blood pressure in 11 distinct studies. We were unable to include results from many studies, $111341565765-6769$ owing to overlap with two large studies (IDACO ${ }^{61}$ and ABP-International ${ }^{64}$ ), which combined results across cohorts.

Three studies examined daytime variability in systolic blood pressure and all cause mortality, of which two were included in the main analysis (fig 5; hazard ratio $1.10,95 \%$ confidence interval 1.04 to 1.16 ). Four studies examined daytime variability in blood pressure and cardiovascular disease mortality, and analysis of three studies with low risk of bias showed a significant association (see supplementary figure e5, 1.12, 1.03 to 1.21). Daytime blood pressure variability was also significantly associated with increased risk of stroke (see supplementary figure e6; 1.11, 1.01 to 1.21). Results for all three outcomes were unchanged in secondary analysis including results from all studies.

No associations were found between variability in blood pressure and cardiovascular disease (see supplementary figure e7) or coronary heart disease events (see supplementary figure e8), although results became significant in secondary analyses. The supplementary file details the results for night-time and 24 hour systolic ambulatory blood pressure (see figures e9 to e18).

\section{Publication bias}

There was no evidence of publication bias for any outcome in relation to long term, mid-term, or short term variability in systolic blood pressure as judged by Egger's test. Significant findings for clinic monitoring would remain unchanged for all outcomes even if at least 20 null effect studies were included in metaanalyses, except for myocardial infarction where only a single null effect study would be required. Results for home monitoring would become non-significant after the addition of a single null effect study and those for variability in ambulatory blood pressure would become non-significant by the addition of between one and six null effect studies, depending on outcome and period of measurement.

\section{Discussion}

This review has systematically assessed the literature for the association of long term (clinic monitoring), mid-term (home monitoring), and short term (ambulatory) variability in blood pressure with cardiovascular outcomes and mortality. Long term variability in measurements is significantly associated with all cause and cardiovascular disease mortality, cardiovascular disease events, stroke, and myocardial infarction, independent of mean blood pressure. Mid-term and short term variability are also associated with mortality, and limited data for other outcomes also broadly support an association with cardiovascular outcomes. Across all analyses (long term, mid-term, and short term), the hazard ratios for coronary heart disease events were smaller than those for stroke, suggesting that the effect 


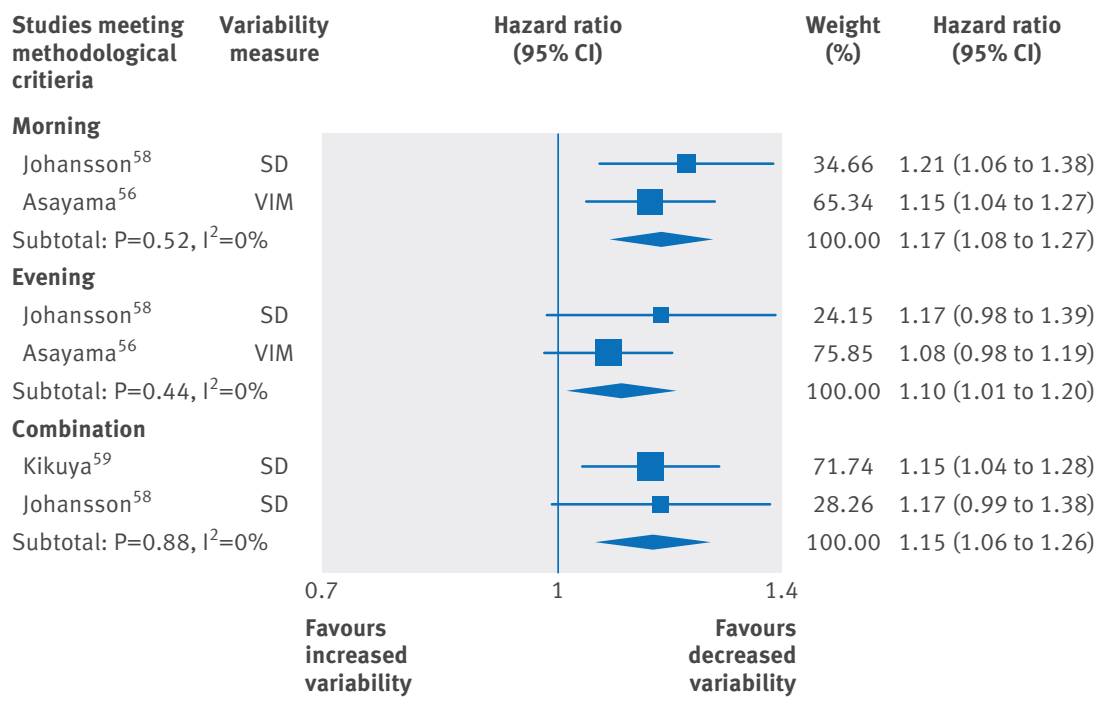

Fig 4 | Random effects meta-analysis of standardized hazard ratios for increases in home systolic blood pressure variability and all cause mortality. SD=standard deviation; $\mathrm{VIM}=$ variation independent of mean

\begin{tabular}{|c|c|c|c|c|}
\hline Study & $\begin{array}{c}\text { Variability } \\
\text { measure }\end{array}$ & $\begin{array}{c}\text { Hazard ratio } \\
(95 \% \mathrm{Cl})\end{array}$ & $\begin{array}{c}\text { Weight } \\
\text { (\%) }\end{array}$ & $\begin{array}{c}\text { Hazard ratio } \\
(95 \% \mathrm{Cl})\end{array}$ \\
\hline
\end{tabular}

Studies meeting methodological critieria $\begin{array}{lc}\text { Hansen }^{61} & \text { ARV } \\ \text { Palatini }^{64} & \text { SD }\end{array}$

Subtotal: $P=0.85,\left.\right|^{2}=0 \%$

Studies not meeting methodological criteria Mancia $^{62}$ SD

Subtotal

Overall: $P=0.95, I^{2}=0 \%$

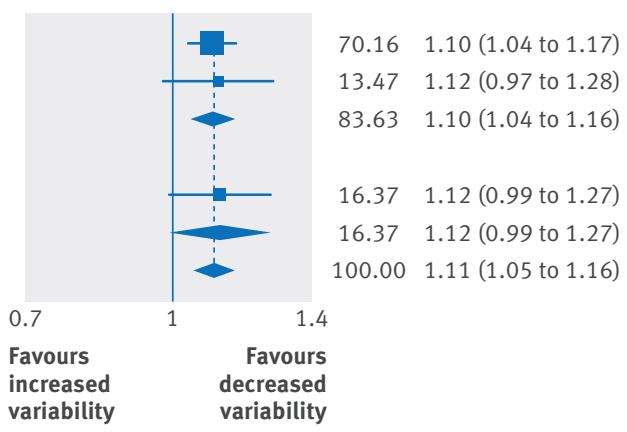

Fig 5 | Random effects meta-analysis of standardised hazard ratios for increases in variability of ambulatory systolic blood pressure and all cause mortality. SD=standard deviation; $A R V=$ average real variability in primary studies, and hence combined more data. For example, our meta-analysis for long term variability and stroke events includes 14 studies, more than double the number in previous analyses. ${ }^{24} 25$ This review also had sufficient data for meta-analysis of the effect of short term variability of blood pressure on outcomes, which was a limitation of a previous work. ${ }^{28}$

Finally, we demonstrated the robustness of results to possible unpublished null effect studies across long term, mid-term, and short term variability in blood pressure. Although results for long term variability may be considered conclusive, results for mid-term and short term variability are more susceptible to publication bias and may warrant further investigation.

\section{Limitations of this review}

Included studies were primarily in older adults (mean age 48.5 to 77 years) and those at increased risk of cardiovascular disease (eg, due to hypertension) and conducted in European or East Asian populations. Hence the applicability of our findings to younger or healthier people and other ethnic groups is unknown. Studies in patients with a history of cerebrovascular events reported the largest hazard ratios, but significant associations remained after removal of these studies from analyses, and so findings remain applicable to people free from cerebrovascular disease. In studies in hypertensive patients, ${ }^{144069}$ blood pressure variability could be confounded by entry criteria (regression to the mean $)^{71}$ and treatment. However, such effects would diminish rather than exaggerate hazard ratios for variability, and so our overall conclusions are sound.

Lack of data from distinct cohorts prevented formal meta-analyses for many outcomes for to mid-term variability in blood pressure. A previous review was similarly limited by paucity of data, ${ }^{26}$ despite broader inclusion criteria. Our meta-analyses for short term variability in blood pressure were also dominated by two large studies. Despite these caveats, results supported an effect of shorter term variability on cardiovascular outcomes, and pooled hazard ratios were similar to those observed for long term variability. We were unable to determine if findings varied with timing and frequency of measurement.

In several analyses, there was significant heterogeneity between studies, potentially due to outlying studies in specific populations (eg, previous vascular disease) or to approximations necessary during data extraction, such as conversion from categorical (eg, from 10 ths ${ }^{10}$ or thirds ${ }^{49}$ ) to continuous scale. However, not all converted hazard ratios were outliers, ${ }^{50}$ and we verified our conversion method in simulated data (not shown). Significant heterogeneity was reduced by removal of outlier studies, but this did not significantly alter the results.

In some cases, few studies contributed to main analyses, and the validity of these meta-analyses is debateable. Secondary analysis utilising data from all studies regardless of quality greatly increased the amount of available data but did not materially change results. Only three otherwise eligible studies 
failed to contribute any quantitative data, despite contact with authors. 444852

In general, there was poor reporting of study factors that may confound the relation between blood pressure variability and outcomes. Although studies were excluded from main analyses based on the three most important factors (prespecified), it was not feasible to do this for all factors. Further adjustment for confounders might be possible using individual patient data but was beyond the scope of this review. The importance of consideration and reporting of such confounding factors in future work on blood pressure variability (and variability in other biological measures) should be emphasised. Although our results indicate that these may be less important in the assessment of blood pressure variability, they may prove instrumental in other clinical areas.

\section{Clinical implications}

The mechanism linking blood pressure variability to cardiovascular events is not well understood. Short term variability in blood pressure is affected by behavioural, emotional, and postural influences on cardiovascular physiology and cardiac rhythm. ${ }^{7273}$ Arterial stiffness contributes to both short term ${ }^{74} 75$ and long term variability in blood pressure..$^{737677}$ Meanwhile, poor control of blood pressure resulting in changes to antihypertensive drugs also affects variability. ${ }^{72}$ Use of certain classes of antihypertensive drugs has also been linked with increased visit-to-visit variability ${ }^{78}$ and may not be entirely explained by adherence. ${ }^{79}$

The estimated standardised hazard ratio for the effect of long term variability in blood pressure on cardiovascular disease mortality was 1.18 . For comparison, the effect of mean blood pressure on cardiovascular disease mortality reported in a previous meta-analysis ${ }^{3}$ corresponds to a standardised hazard ratio of approximately 1.7 (assuming a between person standard deviation of $15 \mathrm{~mm} \mathrm{Hg}$ ). Note that the latter standardised hazard ratio for mean blood pressure is not adjusted for variability, whereas the former (for blood pressure variability) is adjusted for mean blood pressure, showing the additional prognostic value of variability over and above the mean. This supports the results of recent work showing the improved discrimination of models including short term night-time variability in blood pressure ${ }^{64}$ or long term variability, ${ }^{39}$ over and above traditional risk factors.

How does blood pressure variability compare with other risk factors for cardiovascular disease? A recent review $^{80}$ found that the adjusted standardised hazard ratio for increases in cholesterol on cardiovascular disease events varied between 1.16 and 1.29 in primary prevention groups, depending on the measure of cholesterol considered (eg, total cholesterol, triglycerides). Hence variability in blood pressure has similar prognostic value to cholesterol measures (standardised hazard ratio for long term variability on cardiovascular disease events=1.18).

Variability in blood pressure is not easily assessed clinically, and it is unclear if certain measures of variability should be preferred. Some measures could be calculated by hand (eg, average real variability), whereas others could be automatically calculated by electronic health records. This would enable doctors to account for both mean and variability in blood pressure concurrently when assessing cardiovascular risk. For example, assuming a standard deviation for variability (standard deviation) in systolic blood pressure of $5 \mathrm{~mm} \mathrm{Hg}$, an individual with variable blood pressure readings (139, 132, and $125 \mathrm{~mm} \mathrm{Hg}$, mean 132, SD 7) could be considered at $18 \%$ greater risk of cardiovascular disease events than a similar person with stable blood pressure (134, 130, and $132 \mathrm{~mm} \mathrm{Hg}$, mean 132, SD 2). This may be particularly important for patients with a highly variable but comparatively low mean blood pressure or for whom traditional cardiovascular risk estimates lie close to treatment thresholds. Further work is needed to determine the feasibility of obtaining such additional information, and the clinical impact on subsequent risk management.

\section{Conclusion}

Long term variability in blood pressure measured in adults at clinic visits is associated with cardiovascular and mortality outcomes, over and above the effect of mean blood pressure. Mid-term (home monitoring) and short term (ambulatory monitoring) variability in blood pressure is also associated with all cause mortality, but the association with cardiovascular disease outcomes requires further investigation in novel cohorts.

We thank Derek Shaw and David Yeomans, patient representatives, for their contribution to the design and content of the National Institute for Health Research (NIHR) programme grant from which this work arose. The protocol for this review has been published (www.crd.york. ac.uk/PROSPERO/ CRD42014015695).

Contributors: SS, SW, RS, RM, and PG designed the study. SS, SW, KL, KC, and RM screened articles. SS, SW, KL, KC, RS and RM extracted data. SS, KC, and RS carried out statistical analyses. SS drafted the original manuscript and all authors revised the paper. SS is the guarantor.

Funding This manuscript presents independent research commissioned by the NIHR under its programme grants for applied research funding scheme (grant No RP-PG-1209-10051).

Competing interests: All authors have completed the ICMJE uniform disclosure form at www.icmje.org/coi_disclosure.pdf (available on request from the corresponding author) and declare: SS is funded by the National Institute for Health Research School for Primary Care Research (NIHR SPCR), RM has received grants and personal fees from Omron and grants from Lloyds Pharmacy, outside the submitted work; PG reports grants from National Heart Foundation, Australia, outside the submitted work, no other relationships or activities that could appear to have influenced the submitted work. The views expressed are those of the authors and not necessarily those of the NHS, the NIHR, or the Department of Health.

Ethical approval: Not required.

Data sharing: Data extracted from the studies in this review are available on request from the corresponding author.

Transparency: The lead author (SS) affirms that the manuscript is an honest, accurate, and transparent account of the study being reported; that no important aspects of the study have been omitted; and that any discrepancies from the study as planned (and, if relevant, registered) have been explained.

This is an Open Access article distributed in accordance with the terms of the Creative Commons Attribution (CC BY 3.0) license, which permits others to distribute, remix, adapt and build upon this work, for commercial use, provided the original work is properly cited. See: http://creativecommons.org/licenses/by/3.0/. 
1 Lim SS, Vos T, Flaxman AD, et al. A comparative risk assessment of burden of disease and injury attributable to 67 risk factors and risk factor clusters in 21 regions, 1990-2010: a systematic analysis for the Global Burden of Disease Study 2010. Lancet 2012;380:222460. doi:10.1016/S0140-6736(12)61766-8.

2 Law MR, Morris JK, Wald NJ. Use of blood pressure lowering drugs in the prevention of cardiovascular disease: meta-analysis of 147 randomised trials in the context of expectations from prospective epidemiological studies. BMJ 2009;338:b1665. doi:10.1136/bmj. b1665.

3 Lewington S, Clarke R, Qizilbash N, Peto R, Collins R. Prospective Studies Collaboration. Age-specific relevance of usual blood pressure to vascular mortality: a meta-analysis of individual data for one million adults in 61 prospective studies. Lancet 2002;360:1903-13. doi:10.1016/S0140-6736(02)11911-8.

4 Conen D, Bamberg F. Noninvasive 24-h ambulatory blood pressure and cardiovascular disease: a systematic review and meta-analysis. J Hypertens 2008;26:1290-9. doi:10.1097/HJH.0b013e3282f97854.

5 Ward AM, Takahashi O, Stevens R, Heneghan C. Home measurement of blood pressure and cardiovascular disease: systematic review and meta-analysis of prospective studies. J Hypertens 2012;30:449-56. doi:10.1097/HJH.0b013e32834e4aed.

6 Grassi G, Bombelli M, Brambilla G, Trevano FQ, Dell'oro R, Mancia G. Total cardiovascular risk, blood pressure variability and adrenergic overdrive in hypertension: evidence, mechanisms and clinical implications. Curr Hypertens Rep 2012;14:333-8. doi:10.1007/ s11906-012-0273-8.

7 Takahashi O, Glasziou PP, Perera R, Shimbo T, Fukui T. Blood pressure re-screening for healthy adults: what is the best measure and interval? J Hum Hypertens 2012;26:540-6. doi:10.1038/jhh.2011.72.

8 Frattola A, Parati G, Cuspidi C, Albini F, Mancia G. Prognostic value of 24-hour blood pressure variability. J Hypertens 1993;11:1133-7. doi:10.1097/00004872-199310000-00019.

9 Grove JS, Reed DM, Yano K, Hwang LJ. Variability in systolic blood pressure-a risk factor for coronary heart disease? Am J Epidemiol 1997;145:771-6. doi:10.1093/oxfordjournals.aje.a009169.

10 Rothwell PM, Howard SC, Dolan E, et al. Prognostic significance of visit-to-visit variability, maximum systolic blood pressure, and episodic hypertension. Lancet 2010;375:895-905. doi:10.1016/ S0140-6736(10)60308-X

11 Björklund K, Lind L, Zethelius B, Berglund L, Lithell H. Prognostic significance of 24-h ambulatory blood pressure characteristics for cardiovascular morbidity in a population of elderly men. J Hypertens 2004:22:1691-7. doi:10.1097/00004872-200409000-00012.

12 Gavish B, Ben-Dov IZ, Kark JD, Mekler J, Bursztyn M. The association of a simple blood pressure-independent parameter derived from ambulatory blood pressure variability with short-term mortality. Hypertens Res 2009:32:488-95. doi:10.1038/hr.2009.39.

13 Kikuya M, Hozawa A, Ohokubo T, et al. Prognostic significance of blood pressure and heart rate variabilities: the Ohasama study. Hypertension 2000;36:901-6. doi:10.1161/01.HYP.36.5.901.

14 Kawai T, Ohishi M, Ito N, et al. Alteration of vascular function is an important factor in the correlation between visit-to-visit blood pressure variability and cardiovascular disease. J Hypertens 2013;31:1387-95, discussion 1395. doi:10.1097/HJH.0b013e328360f796.

15 Gao S, Hendrie HC, Wang C, et al. Redefined blood pressure variability measure and its association with mortality in elderly primary care patients. Hypertension 2014:64:45-52. doi:10.1161/HYPERTENSIONAHA.114.03576.

16 Lau K-K, Wong Y-K, Teo K-C, et al. Long-term prognostic implications of visit-to-visit blood pressure variability in patients with ischemic stroke. Am J Hypertens 2014:27:1486-94. doi:10.1093/ajh/hpu070.

17 Lévesque LE, Hanley JA, Kezouh A, Suissa S. Problem of immortal time bias in cohort studies: example using statins for preventing progression of diabetes. BMJ 2010;340:b5087. doi:10.1136/bmj.b5087.

18 Suchy-Dicey AM, Wallace ER, Mitchell SVE, et al. Blood pressure variability and the risk of all-cause mortality, incident myocardia infarction, and incident stroke in the cardiovascular health study. Am J Hypertens 2013;26:1210-7. doi:10.1093/aih/hpt092.

19 Wei Y, Jin Z, Shen G, et al. Effects of intensive antihypertensive treatment on Chinese hypertensive patients older than 70 years. J Clin Hypertens (Greenwich) 2013;15:420-7. doi:10.1111/jch.12094.

20 Pickering TG, Harshfield GA, Kleinert HD, Blank S, Laragh JH. Blood pressure during normal daily activities, sleep, and exercise. Comparison of values in normal and hypertensive subjects. JAMA 1982;247:992-6. doi:10.1001/jama.1982.03320320028025.

21 Modesti PA, Morabito M, Bertolozzi I, et al. Weather-related changes in 24-hour blood pressure profile: effects of age and implications for hypertension management. Hypertension 2006;47:155-61. doi:10.1161/01.HYP.0000199192.17126.d4.

22 Lane D, Beevers M, Barnes N, et al. Inter-arm differences in blood pressure: when are they clinically significant? J Hypertens 2002;20: 1089-95. doi:10.1097/00004872-200206000-00019.

23 Linfors EW, Feussner JR, Blessing CL, Starmer CF, Neelon FA, McKee PA. Spurious hypertension in the obese patient. Effect of sphygmomanometer cuff size on prevalence of hypertension. Arch Intern Med 1984;144:1482-5. doi:10.1001/archinte.1984.00350190188032.
24 Diaz KM, Tanner RM, Falzon L, et al. Visit-to-visit variability of blood pressure and cardiovascular disease and all-cause mortality: a systematic review and meta-analysis. Hypertension 2014;64:965-82 doi:10.1161/HYPERTENSIONAHA.114.03903.

25 Tai C, Sun Y, Dai N, et al. Prognostic significance of visit-to-visit systolic blood pressure variability: a meta-analysis of 77,299 patients. J Clin Hypertens (Greenwich) 2015;17:107-15. doi:10.1111/jch.12484.

26 Stergiou GS, Ntineri A, Kollias A, Ohkubo T, Imai Y, Parati G. Blood pressure variability assessed by home measurements: a systematic review. Hypertens Res 2014:37:565-72. doi:10.1038/hr.2014.2.

27 Manning LS, Rothwell PM, Potter JF, Robinson TG. Prognostic Significance of Short-Term Blood Pressure Variability in Acute Stroke: Systematic Review. Stroke 2015;46:2482-90. doi:10.1161/STROKEAHA.115.010075.

28 Taylor KS, Heneghan CJ, Stevens RJ, Adams EC, Nunan D, Ward A. Heterogeneity of prognostic studies of 24 -hour blood pressure variability: systematic review and meta-analysis. PLoS One 2015:10:e0126375. doi:10.1371/journal.pone.0126375.

29 Daugirdas JT. Pathophysiology of dialysis hypotension: an update. Am J Kidney Dis 2001;38(Suppl 4):S11-7. doi:10.1053/ajkd.2001.28090.

30 Inrig JK. Intradialytic hypertension: a less-recognized cardiovascular complication of hemodialysis. Am J Kidney Dis 2010;55:580-9. doi:10.1053/j.ajkd.2009.08.013

31 Inrig JK, Oddone EZ, Hasselblad V, et al. Association of intradialytic blood pressure changes with hospitalization and mortality rates in prevalent ESRD patients. Kidney Int 2007;71:454-61. doi:10.1038/sj.ki.5002077.

32 Inrig JK, Patel UD, Toto RD, Szczech LA. Association of blood pressure increases during hemodialysis with 2-year mortality in incident hemodialysis patients: a secondary analysis of the Dialysis Morbidity and Mortality Wave 2 Study. Am J Kidney Dis 2009;54:881-90. doi:10.1053/i.ajkd.2009.05.012

33 National Institute for Health and Care Excellence. Hypertension: Clinical management of primary hypertension in adults (NICE CG127) National Institute for Health and Care Excellence, 2011.

34 Bring J. How to standardize regression coefficients. Am Stat 1994: 48:209-13.

35 Hayden JA, van der Windt DA, Cartwright JL, Côté P, Bombardier C. Assessing bias in studies of prognostic factors. Ann Intern Med 2013;158:280-6. doi:10.7326/0003-4819-158-4-201302190-00009.

36 Egger M, Davey Smith G, Schneider M, Minder C. Bias in meta-analysis detected by a simple, graphical test. BMJ 1997;315:629-34. doi:10.1136/bmi.315.7109.629.

37 Rosenberg MS. The file-drawer problem revisited: a general weighted method for calculating fail-safe numbers in meta-analysis. Evolution 2005;59:464-8. doi:10.1111/j.0014-3820.2005.tb01004.x.

38 Arashi H, Ogawa H, Yamaguchi J, Kawada-Watanabe E, Hagiwara N. Impact of visit-to-visit variability and systolic blood pressure control on subsequent outcomes in hypertensive patients with coronary artery disease (from the HII-CREATE substudy). Am / Cardiol 2015;116:236-42. doi:10.1016/j.amjcard.2015.04.011.

39 Blacher J, Safar ME, Ly C, Szabo de Edelenyi F, Hercberg S, Galan P. Blood pressure variability: cardiovascular risk integrator or independent risk factor? J Hum Hypertens 2015;29:122-6. doi:10.1038/jhh.2014.44.

40 Carr MJ, Bao Y, Pan J, Cruickshank K, McNamee R. The predictive ability of blood pressure in elderly trial patients. J Hypertens 2012;30:172533. doi:10.1097/HJH.0b013e3283568a73.

41 Eguchi K, Hoshide S, Schwartz JE, Shimada K, Kario K. Visit-to-visit and ambulatory blood pressure variability as predictors of incident cardiovascular events in patients with hypertension. Am J Hypertens 2012;25:962-8. doi:10.1038/ajh.2012.75.

42 Hara A, Thijs L, Asayama K, Jacobs L, Wang JG, Staessen JA Randomised double-blind comparison of placebo and active drugs for effects on risks associated with blood pressure variability in the Systolic Hypertension in Europe trial. PLoS One 2014;9:e103169. doi:10.1371/journal.pone.0103169.

43 Hata J, Arima H, Rothwell PM, et al. ADVANCE Collaborative Group. Effects of visit-to-visit variability in systolic blood pressure on macrovascular and microvascular complications in patients with type 2 diabetes mellitus: the ADVANCE trial. Circulation 2013:128:1325-34 doi:10.1161/CIRCULATIONAHA.113.002717.

44 Hsieh Y-T, Tu S-T, Cho T-J, Chang SJ, Chen JF, Hsieh MC. Visit-to-visit variability in blood pressure strongly predicts all-cause mortality in patients with type 2 diabetes: a 5.5-year prospective analysis. Eur I Clin Invest 2012;42:245-53. doi:10.1111/j.1365-2362.2011.02574.x.

45 Kostis JB, Sedjro JE, Cabrera J, et al. Visit-to-visit blood pressure variability and cardiovascular death in the Systolic Hypertension in the Elderly Program. J Clin Hypertens (Greenwich) 2014;16:34-40. doi:10.1111/jch.12230

46 Lau KK, Wong YK, Chan YH, et al. Visit-to-visit blood pressure variability as a prognostic marker in patients with cardiovascular and cerebrovascular diseases-relationships and comparisons with vascular markers of atherosclerosis. Atherosclerosis 2014;235:230-5. doi:10.1016/j.atherosclerosis.2014.04.015.

47 Mallamaci F, Minutolo R, Leonardis D, et al. Long-term visit-to-visit office blood pressure variability increases the risk of adverse cardiovascular outcomes in patients with chronic kidney disease. Kidney Int 2013;84:381-9. doi:10.1038/ki.2013.132. 
48 Mancia G, Facchetti R, Parati G, Zanchetti A. Visit-to-visit blood pressure variability in the European Lacidipine Study on Atherosclerosis: methodological aspects and effects of antihypertensive treatment. J Hypertens 2012;30:1241-51. doi:10.1097/HJH.0b013e32835339ac.

49 McMullan CJ, Bakris GL, Phillips RA, Forman JP. Association of BP variability with mortality among African Americans with CKD. Clin J Am Soc Nephrol 2013;8:731-8. doi:10.2215/CJN.10131012.

50 Muntner P, Whittle J, Lynch Al, et al. Visit-to-Visit Variability of Blood Pressure and Coronary Heart Disease, Stroke, Heart Failure, and Mortality: A Cohort Study. Ann Intern Med 2015;163:329-38. doi:10.7326/M14-2803.

51 Poortvliet RKE, Ford I, Lloyd SM, et al. Blood pressure variability and cardiovascular risk in the PROspective Study of Pravastatin in the Elderly at Risk (PROSPER). PLoS One 2012;7:e52438. doi:10.1371/ journal.pone.0052438

52 Rossignol P, Girerd N, Gregory D, Massaro J, Konstam MA, Zannad F. Increased visit-to-visit blood pressure variability is associated with worse cardiovascular outcomes in low ejection fraction heart failure patients: Insights from the HEAAL study. Int J Cardiol 2015;187:183-9. doi:10.1016/j.ijcard.2015.03.169.

53 Shimbo D, Newman JD, Aragaki AK, et al. Association between annual visit-to-visit blood pressure variability and stroke in postmenopausal women: data from the Women's Health Initiative. Hypertension 2012:60:625-30 doi:10.1161/HYPERTENSIONAHA.112.193094.

54 Yu JM, Kong QY, Schoenhagen P, et al. The prognostic value of long-term visit-to-visit blood pressure variability on stroke in real-world practice: a dynamic cohort study in a large representative sample of Chinese hypertensive population. Int J Cardiol 2014;177:995-1000. doi:10.1016/j.ijcard.2014.09.149.

55 Rakugi H, Ogihara T, Saruta T, et al. COLM Investigators. Preferable effects of olmesartan/calcium channel blocker to olmesartan/diuretic on blood pressure variability in very elderly hypertension: COLM study subanalysis. J Hypertens 2015;33:2165-72. doi:10.1097/HJH.0000000000000668.

56 Asayama K, Kikuya M, Schutte R, et al. Home blood pressure variability as cardiovascular risk factor in the population of Ohasama. Hypertension 2013;61:61-9. doi:10.1161/HYPERTENSIONAHA.111.00138.

57 Hashimoto T, Kikuya M, Ohkubo T, et al. Home blood pressure level, blood pressure variability, smoking, and stroke risk in Japanese men: the Ohasama study. Am J Hypertens 2012;25:883-91. doi:10.1038/ajh.2012.62

58 Johansson JK, Niiranen TJ, Puukka PJ, Jula AM. Prognostic value of the variability in home-measured blood pressure and heart rate: the Finn-Home Study. Hypertension 2012;59:212-8. doi:10.1161/ HYPERTENSIONAHA.111.178657.

59 Kikuya M, Ohkubo T, Metoki H, et al. Day-by-day variability of blood pressure and heart rate at home as a novel predictor of prognosis: the Ohasama study. Hypertension 2008;52:1045-50. doi:10.1161/ HYPERTENSIONAHA.107104620.

60 Gavish B, Bursztyn M. Blood pressure and heart period variability ratios derived from 24-h ambulatory measurements are predictors of all-cause mortality. J Hypertens 2015;33:491-8, discussion 498. doi:10.1097/HJH.0000000000000459.

61 Hansen TW, Thijs L, Li Y, et al. International Database on Ambulatory Blood Pressure in Relation to Cardiovascular Outcomes Investigators. Prognostic value of reading-to-reading blood pressure variability over 24 hours in 8938 subjects from 11 populations. Hypertension 2010;55:1049-57. doi:10.1161/HYPERTENSIONAHA.109.140798.

62 Mancia G, Bombelli M, Facchetti R, et al. Long-term prognostic value of blood pressure variability in the general population: results of the Pressioni Arteriose Monitorate e Loro Associazioni Study. Hypertension 2007:49:1265-70. doi:10.1161/HYPERTENSIONAHA.107.088708.

63 Mena LJ, Maestre GE, Hansen TW, et al. International Database on Ambulatory Blood Pressure in Relation to Cardiovascular Outcomes (IDACO) Investigators. How many measurements are needed to estimate blood pressure variability without loss of prognostic information? Am J Hypertens 2014;27:46-55. doi:10.1093/ajh/hpt142.
64 Palatini P, Reboldi G, Beilin LJ, et al. Added predictive value of night-time blood pressure variability for cardiovascular events and mortality: the Ambulatory Blood Pressure-International Study. Hypertension 2014;64:487-93. doi:10.1161/HYPERTENSIONAHA.114.03694

65 Pierdomenico SD, Di Nicola M, Esposito AL, et al. Prognostic value of different indices of blood pressure variability in hypertensive patients. Am J Hypertens 2009;22:842-7. doi:10.1038/ajh.2009.103.

66 Pierdomenico SD, Lapenna D, Di Tommaso R, et al. Blood pressure variability and cardiovascular risk in treated hypertensive patients. Am J Hypertens 2006:19:991-7. doi:10.1016/j.amjhyper.2006.03.009.

67 Pierdomenico SD, Lapenna D, Bucci A, et al. Blood pressure variability and prognosis in uncomplicated mild hypertension. Am Heart J 2005;149:934-8. doi:10.1016/j.ahj.2004.06.029.

68 Pringle E, Phillips C, Thijs L, et al. Syst-Eur investigators. Systolic blood pressure variability as a risk factor for stroke and cardiovascular mortality in the elderly hypertensive population. J Hypertens 2003;21:2251-7. doi:10.1097/00004872-200312000-00012.

69 Verdecchia P, Angeli F, Gattobigio R, Rapicetta C, Reboldi G. Impact of blood pressure variability on cardiac and cerebrovascular complications in hypertension. Am J Hypertens 2007;20:154-61. doi:10.1016/j.amjhyper.2006.07.017.

70 Farrell B, Godwin J, Richards S, Warlow C. The United Kingdom transient ischaemic attack (UK-TIA) aspirin trial: final results. J Neurol Neurosurg Psychiatry 1991;54:1044-54. doi:10.1136/jnnp.54.12.1044.

71 Yudkin PL, Stratton IM. How to deal with regression to the mean in intervention studies. Lancet 1996;347:241-3. doi:10.1016/ S0140-6736(96)90410-9.

72 Parati G, Ochoa JE, Lombardi C, Bilo G. Assessment and management of blood-pressure variability. Nat Rev Cardiol 2013;10:143-55. doi:10.1038/nrcardio.2013.1.

73 Mancia G. Short- and long-term blood pressure variability: present and future. Hypertension 2012;60:512-7. doi:10.1161/ HYPERTENSIONAHA.112.194340.

74 García-García Á, García-Ortiz L, Recio-Rodríguez II, et al. Relationship of 24-h blood pressure variability with vascular structure and function in hypertensive patients. Blood Press Monit 2013;18:101-6. doi:10.1097/MBP.0b013e32835ebc58.

75 Schillaci G, Bilo G, Pucci G, et al. Relationship between short-term blood pressure variability and large-artery stiffness in human hypertension: findings from 2 large databases. Hypertension 2012:60:369-77. doi:10.1161/HYPERTENSIONAHA.112.197491.

76 Nagai M, Hoshide S, Ishikawa J, Shimada K, Kario K. Visit-to-visit blood pressure variations: new independent determinants for carotid artery measures in the elderly at high risk of cardiovascular disease. J Am Soc Hypertens 2011;5:184-92. doi:10.1016/j.jash.2011.03.001.

77 Okada H, Fukui M, Tanaka M, et al. Visit-to-visit variability in systolic blood pressure is correlated with diabetic nephropathy and atherosclerosis in patients with type 2 diabetes. Atherosclerosis 2012;220:155-9. doi:10.1016/j.atherosclerosis.2011.10.033.

78 Webb AJ, Fischer U, Mehta Z, Rothwell PM. Effects of antihypertensivedrug class on interindividual variation in blood pressure and risk of stroke: a systematic review and meta-analysis. Lancet 2010;375:90615. doi:10.1016/S0140-6736(10)60235-8

79 Muntner P, Levitan EB, Joyce C, et al. Association between antihypertensive medication adherence and visit-to-visit variability of blood pressure. J Clin Hypertens (Greenwich) 2013;15:112-7. doi:10.1111/jch.12037.

80 Perera R, Clarke P, Farmer A, et al. Optimal strategies for monitoring lipid levels in patients at risk or with cardiovascular disease: a systematic review with statistical and cost-effectiveness modelling. Health Technol Assess 2015;19:1-401. doi:10.3310/hta191000.

(c) BMJ Publishing Group Ltd 2016

Supplementary information: supplementary tables e1-e7 and figures e1-e18 